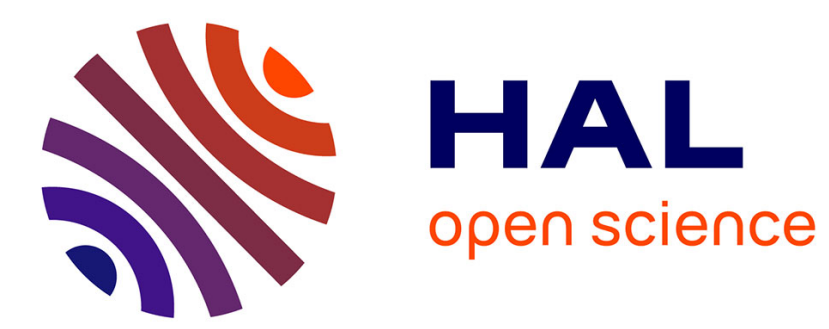

\title{
Les services secrets gaullistes à l'épreuve de la politique (1940-1947)
}

Sebastien Laurent

\section{To cite this version:}

Sebastien Laurent. Les services secrets gaullistes à l'épreuve de la politique (1940-1947). Politix, A paraître, 14 (54), pp.139-153. 10.3406/polix.2001.1158 . hal-02496699

\section{HAL Id: hal-02496699 \\ https://hal.science/hal-02496699}

Submitted on 3 Mar 2020

HAL is a multi-disciplinary open access archive for the deposit and dissemination of scientific research documents, whether they are published or not. The documents may come from teaching and research institutions in France or abroad, or from public or private research centers.
L'archive ouverte pluridisciplinaire HAL, est destinée au dépôt et à la diffusion de documents scientifiques de niveau recherche, publiés ou non, émanant des établissements d'enseignement et de recherche français ou étrangers, des laboratoires publics ou privés.

\section{(이) $\$$}

Distributed under a Creative Commons Attribution - NonCommercial - NoDerivatives| 4.0 


\begin{abstract}
The Politicization of French Intelligence Services: From Military to Political Control

(1940-1947)

Sébastien Laurent

The exceptional military and political events due to the World War II have deeply modified the conditions under which the French intelligence services acted beforehand. The strict partition between military and political fields at the time of the third Republic has to be considered ail over again. The purpose of this article is to analyse the reasons of this drastic upheaval consisting in the intelligence services escaping from the military command. In London, then in Algiers there was a series of conflicts between the inner French Resistance and the de Gaulle's attendants, leading to the foundation of the SDECE (Service de documentation exterieure et de contre-espionnage). Hereafter the intelligence services, linked since 1945 to those of the Presidence du conseil became the aims of hard political struggles in parliamentary circles as in the press of the new regime. The "Passy affair" which occurred in 1946 appeared as a typical example of the new balanced political and journalistic influences from which since then, the secret officers could not escape.
\end{abstract}

\title{
Résumé
}

Les services secrets gaullistes à l'épreuve de la politique (1940-1947)

Sébastien Laurent

Les circonstances militaires et politiques exceptionnelles crées par la Seconde guerre mondiale ont profondément modifié les conditions dans lesquelles les services de renseignement militaire agissaient auparavant. La stricte séparation entre le militaire et le politique sous la troisième République a ainsi été entièrement remise en cause. Cette étude a pour objet d'analyser les raisons de cette révolution qui voit les services de renseignement militaire sortir du " pré carré » militaire. A Londres puis à Alger, se jouent entre la Résistance intérieure et l'entourage du général de Gaulle, les principales étapes de conflits présidant à la naissance du SDECE. Rattachés à la présidence du Conseil à partir de 1945, les services sont désormais l'enjeu de luttes politiques très vives, dans les assemblées politiques et dans la presse du nouveau régime. L'affaire Passy qui surgit en 1946 est un exemple caractéristique des nouveaux équilibres politiques et médiatiques auxquels sont désormais soumis les officiers de l'ombre. 


\section{Les services secrets gaullistes à l'épreuve de la politique (1940-1947)}

Sébastien LAURENT

11 7 out au long de la III ${ }^{e}$ République, les services de renseignement militaires ont fonctionné sur une organisation héritée de l'Affaire - Dreyfus et de la Grande Guerre ${ }^{1}$. S'exerçant dans le cadre républicain, leur activité était fondée sur une rigoureuse distinction entre sphère militaire et sphère politique. Pendant cette période, les services de renseignement militaires n'ont relevé hiérarchiquement que de la seule institution militaire et ont été tenus, officiellement, à l'écart de la politique. Par ailleurs, à la suite de l'Affaire Dreyfus, les officiers de renseignement ont quitté, avec soulagement, l'espace public qu'ils avaient investi pour défendre l'honneur de l'armée. Les circonstances politiques et militaires exceptionnelles imposées par la guerre ont profondément modifié, de 1940 à 1945 , les conditions dans lesquelles les officiers de renseignement servaient le gouvernement et l'Etat. De même, la disparition du régime républicain a brouillé les enjeux des relations entre les militaires et la politique : c'est en fait l'ensemble du dispositif de renseignement qui a été ébranlé. Ainsi, les conditions dans lesquelles les services de renseignement ont travaillé pendant le conflit ont pesé très fortement lors de la profonde réorganisation des services dans les dernières années de la guerre avec la création de la

1. Cet article est tiré d'une communication faite au séminaire de doctorat de J.-J. Becker et $P$. Levillain à l'Université de Paris-X-Nanterre en avril 2000. Nous remercions J.-J. Becker de nous avoir autorisé à la reproduire. A cette séance, E. Duhamel était présent. Il a formulé au cours de la discussion des observations et des remarques dont cette version a essayé de tenir compte. 
Direction de la surveillance du territoire (DST) en 1944 et celle du Service de documentation extérieure et de contre-espionnage (SDECE) en 1945.

\section{L'organisation républicaine du renseignement}

Le terme générique de services secrets désigne habituellement l'ensemble des services de renseignement - militaires et politiques - travaillant pour l'Etat. Leur activité, au carrefour de plusieurs fonctions régaliennes, repose sur la nécessité d'informer les organes de décision gouvernementaux de tout ce qui peut mettre en péril l'existence de l'Etat mais également de tout ce qui peut lui permettre de prendre un avantage - de quelque nature qu'il soit vis-à-vis des autres Etats. Si la fonction de renseignement est unique, sa mise en ouvre en revanche fait appel dans ses différentes modalités à deux corps distincts de fonctionnaires. L'organisation traditionnelle des services secrets telle qu'elle existe encore aujourd'hui a été fixée en 1899 à l'occasion de l'Affaire Dreyfus puis définitivement confirmée en 1944-1945. A partir de la fin du XIXe siècle, en temps de paix, l'activité de recherche du renseignement sur le territoire national relevait d'un service civil, la police spéciale, dénommée ensuite "surveillance du territoire ». La même tâche incombait à l'armée, mais à l'extérieur du territoire. Bien que le général de Galliffet soit à l'origine de cette distinction fondatrice, cette répartition des missions entre policiers et militaires est devenue un principe républicain depuis le gouvernement Waldeck-Rousseau. Le comportement de l'état-major, la crainte réactivée du césarisme et l'émergence d'une police républicaine à l'occasion de l'Affaire expliquent en grande partie cette séparation ${ }^{2}$. La division du travail entre policiers et militaires ne souffrait que d'une exception, en temps de guerre, puisque au terme de la loi du 9 août 1849 sur l'état de siège, le contrôle des pouvoirs de police était attribué à l'autorité militaire. Dans ce cadre, les services de renseignements militaires (SR) coiffaient l'ensemble des services de renseignement œuvrant à l'extérieur comme à l'intérieur du territoire et les forces de police étaient alors intégrées au SR.

Au début du XIXe siècle, les missions de renseignements qui étaient confiées à l'armée avaient en théorie pour objet exclusif de tenir à jour l'ordre de bataille ennemi, c'est-à-dire le dispositif de combat des unités adverses. Or, dès le milieu du XIX ${ }^{e}$ siècle, on observe une modification des objectifs du SR : les services secrets militaires, alors embryonnaires au sein du service du Dépôt de la Guerre, étendent leur activité à la recherche des renseignements économiques et politiques. La Grande Guerre sanctionna définitivement cette évolution dans la mesure où le haut commandement et le grand

2. Cf. Berlière (J.-M.), " La généalogie d'un double tradition policière », in Birnbaum (P.), dir., $L a$ France de l'Affaire Dreyfus, Paris, Gallimard, 1994. 
quartier général ont eu besoin de renseignements intéressant l'ensemble de la vie des nations engagées dans le conflit. Le retour à la paix ne s'est pas traduit par une remise en cause de cette évolution. En effet, l'article 2 de $\mathrm{l}^{\prime}$ Instruction provisoire sur la recherche et l'interprétation des renseignements publiée par le ministère de la Guerre en 1922 donne désormais une définition très large et non plus seulement militaire des objectifs du renseignement en les hiérarchisant de façon nouvelle : "Les renseignements s'étendent à tous les domaines de l'activité ennemie (politique, économique, militaire), lorsqu'il s'agit du commandement suprême ${ }^{3}$. "

\section{Une administration apolitique?}

En dépit de cette évolution, les militaires qui ont servi dans les services de renseignements sous la III ${ }^{e}$ République ont toujours tenu un discours reposant sur un double argumentaire - technique et apolitique - dont l'un des objectifs était de nier l'extension des missions qui leur avaient été confiées depuis la première guerre mondiale. Les nombreux ouvrages de Mémoires et les divers plaidoyers pro domo, mais aussi certains écrits théoriques des professionnels du renseignement, sont fortement imprégnés de cette tonalité. Une étude socioculturelle de ce corps à part dans la société militaire montre que les officiers de renseignement se présentent comme des spécialistes du renseignement ou du contre-espionnage et comme des techniciens du secret, défendant l'idée que leur mission est exclusivement militaire. Ceci leur permettait, plus encore que le reste de l'armée, de se situer dans une position de strict apolitisme ${ }^{4}$. Pour certains de ces officiers, qui se trouvaient en liaison avec les autorités politiques, les contraintes de soumission du pouvoir militaire aux autorités politiques s'imposaient plus qu'à d'autres. Or, on remarque que ce discours en quelque sorte préventif est d'autant plus fort que ces officiers se trouvent auprès d'organes de décision politiques, à la différence de leurs camarades affectés dans des unités militaires ou en état-major. L'activité des services de renseignement dans l'entre-deux-guerres conduit à nuancer très fortement ce type de position : dès les années 1920, la surveillance des nationalistes et des groupements révolutionnaires en métropole comme dans $1^{\prime} E m p i r e^{5}$ devient, en collaboration avec la surveillance du territoire, une activité du SR français. Quelques officiers supérieurs et généraux collaborent dans les années 1930 au réseau "Corvignolles", dont l'objectif est de démanteler les cellules

3. Ministère de la Guerre, Instruction provisoire sur la recherche et l'interprétation des renseignements, Paris, Imprimerie nationale, 1922, p. 11.

4. Sur l'ambivalence de cette notion, cf. Forcade (O.), Duhamel (E.), Vial (P.), dir., Militaires en République 1870-1962. Les officiers, le pouvoir et la vie publique en France, Paris, Publications de la Sorbonne, 1999.

5. En 1937, G. Mandel, ministre des Colonies, créa le Service de renseignements intercolonial (SRI), organe composé de militaires. 
communistes dans les unités militaires: si l'existence d'une cagoule militaire n'a aucun fondement réel, on peut en revanche se poser la question de savoir dans quelle mesure certains officiers du SR de l'époque ont pu, à titre individuel, aider ce type d'activité.

Le discours des officiers du SR sur leur identité marginale au sein de l'armée relève d'une réalité particulière: en effet, les quelques officiers de renseignement amenés à se tenir auprès du gouvernement sont tenus à part par leurs camarades d'état-major et par ceux servant d'autres armes, mais ils ne constituent pas pour autant une profession militaire. Le début de la III ${ }^{e}$ République a certes vu la mise en place $d^{\prime}$ une administration militaire nouvelle et d'un dispositif de renseignement avec la création du $2^{e}$ bureau d'une part et de la section de statistiques (qui devint par la suite le SR-SCR) d'autre part, mais ce mouvement bureaucratique ne s'est pas accompagné de la mise en place de filières de formation aux métiers du renseignement ou même d'écoles d'application existant pourtant dans les autres armes. L'une des faiblesses du renseignement militaire sous la IIIe République réside probablement dans le fait que cette nouvelle fonction a été institutionnalisée mais sans pouvoir s'appuyer sur une véritable professionnalisation que l'institution militaire, en partie par méfiance mais aussi par ignorance de l'importance de la fonction de renseignement, n'a jamais voulu encourager6.

La défaite de 1940 bouleversa profondément et durablement le dispositif de renseignement traditionnel. Cette modification intervint à deux niveaux : dans l'articulation des services de renseignement avec l'autorité politique mais également dans la nature des missions confiées aux services. Cette évolution s'inscrivit dans le cadre plus général d'une crise morale profonde de l'armée. Essayant, à l'image de Weygand, de rejeter la responsabilité de la défaite sur le pouvoir politique, l'armée se trouva confrontée au choix entre le respect ou le refus de l'armistice. Dans son immense majorité, l'armée - en métropole comme dans l'Empire - ne fit pas le choix de la poursuite du combat aux côtés du général de Gaulle. Cependant, le pouvoir à Londres était détenu par un officier général qui se dota assez rapidement d'un embryon d'Etat', comprenant notamment un service des renseignements. Deux structures de renseignements français allaient alors coexister: les services de renseignements de l'armée d'armistice, en grande partie

6. Nous renvoyons pour plus de détails à notre contribution : Laurent (S.), "Le service secret de l'Etat. La part des militaires (1870-1945) ", in Baruch (M.-O.), Duclert (V.), Serviteurs de l'Etat. Une histoire politique de l'administration française 1875-1945, Paris, La Découverte, 2000.

7. Nous renvoyons pour cet aspect à l'analyse novatrice de Crémieux-Brilhac (J.-L.), La France libre. De l'appel du 18 juin à la Libération, Paris, Gallimard, 1996. 
clandestins, et les services spéciaux gaullistes, tous deux s'ignorant au début puis entrant en conflit à partir de $1943^{8}$.

\section{La révision des conceptions orthodoxes du général de Gaulle}

A l'origine, le général de Gaulle était imprégné de la vision commune aux officiers formés sous la III République : au sein du mouvement de la France Libre, les questions politiques devaient être tenues à l'écart des structures militaires. Dans une note datée du 8 octobre 1941, remise à Hugh Dalton qui dirigeait le Special Operations Executive (SOE), service d'action clandestine en Europe, il indiquait de façon très nette sa conception : "L'action proprement militaire [...] est actuellement en bonne voie par les services spéciaux de la France Libre en liaison avec les services spéciaux britanniques. Mais il y a lieu, maintenant, d'entreprendre l'action politique, qui est et doit être distincte de l'action militaire et comporter des hommes et des moyens différents ${ }^{9}$." En matière de renseignement, différents témoignages du colonel André Dewavrin («Passy»), à qui il avait confié les services de renseignement, indiquent que le général de Gaulle, fidèle en cela aussi à l'appréciation traditionnelle des officiers non spécialistes, accordait peu $\mathrm{d}^{\prime}$ importance au renseignement ${ }^{10}$.

Malgré ces principes, le général de Gaulle, qui était contraint de faire flèche de tout bois face à la pénurie de moyens, dut outrepasser la séparation entre autorité politique et autorité militaire. Il créa en décembre 1940 une "direction des affaires politiques" confiée à Gaston Palewski (1901-1984). La note de service instituant cette direction passait outre le principe de séparation puisqu'elle stipulait dans son article 3 que le $2^{\mathrm{e}}$ bureau devait coopérer à l'action politique ${ }^{11}$. Cette disposition importante qui allait bien au-delà de l'esprit de l'Instruction provisoire sur la recherche et l'interprétation des renseignements de 1922, indique que dès 1940 le général de Gaulle n'avait d'autre choix que d'associer le militaire au politique. Il y eut ainsi dans la situation difficile de la France libre en 1940 le germe de nombreux développements du pouvoir gaullien.

Dans le domaine du renseignement, l'improvisation et l'aide anglaise tinrent lieu de méthode. A l'été 1940, De Gaulle désigna le capitaine du génie André

\footnotetext{
8. Sur ce point précis, cf. Laurent (S.), "Renseignement militaire et action politique. Le BCRA et les services spéciaux de l'armée d'armistice", in Lacoste (P.), dir., La culture française du renseignement, Paris, Economica, 1998.

9. Note reproduite dans de Gaulle (C.), Mémoires de guerre. L'appel 1940-1942, Paris, Plon, 1954, p. 625.

10. Colonel Passy, Soutenirs I. 2ime Bureau Londres, Monte-Carlo, Raoul Solar éditeur, 1947, p. 145, Service historique de l'armée de terre., $3 \mathrm{~K} 29 \mathrm{I}$, plage 3, entretien avec le colonel Passy. 11. Note de service reproduite dans Palewski (G.), Mémoires d'action 1924-1974, Paris, Plon, 1988 , p. 152-153.
} 
Dewavrin (1911-1998) pour diriger son $2^{\text {e }}$ bureau, transformé en SR en avril 1941, puis appelé Bureau central de renseignement et d'action militaire (BCRAM) en janvier 1942. Cette évolution est, au regard de l'organisation traditionnelle du dispositif de renseignement militaire, une première originalité : le $2^{e}$ bureau, organe de synthèse du renseignement, remplissait des tâches entièrement différentes de celles d'un SR dont la mission exclusive était la recherche du renseignement. Cette dichotomie apparemment technique recouvrait en fait des pratiques, des métiers, mais aussi des types d'officiers profondément différents. L'adjoint de Dewavrin, le capitaine André Manuel (né en 1905), reconnut après la guerre l'inexpérience des services spéciaux gaullistes dans les premiers temps : "On nous a souvent reproché de n'être que des amateurs. C'est parfaitement exact, mais nous ne pouvions pas attendre. Les spécialistes n'arrivèrent que fin 1942 dans le sillage du général Giraud, après le débarquement en Afrique du Nord ${ }^{12}$. " L'aide et les méthodes anglaises furent décisives, celles de l'Intelligence service dans le domaine du renseignement et celles du SOE en ce qui concerne l'action, activité à laquelle le SR-SCR de la III République et les services spéciaux de l'armée d'armistice ne s'étaient pas intéressés. A cet égard, le terme de " services spéciaux » caractérise très justement le BCRAM qui, sous l'influence anglaise, sut s'adapter plus tôt que les services de renseignements classiques ${ }^{13}$ aux nouvelles conditions de la guerre moderne. En 1940-1941, la nature des informations recueillies par le service du capitaine Dewavrin était majoritairement d'ordre militaire, de Gaulle lui ayant interdit de recueillir tout renseignement $d^{\prime}$ ordre politique ${ }^{14}$, activité relevant de la direction des affaires politiques.

Cette dernière disparut en septembre 1941 lorsque le général de Gaulle créa le Comité national français: au sein de cet embryon de gouvernement, André Diethelm (1896-1941), à la tête du commissariat national à l'intérieur (CNI) hérita des attributions de Palewski. Le $2^{\mathrm{e}}$ bureau devenu BCRAM rechigna à coopérer avec le CNI, organisme entièrement civil qui avait le monopole des relations avec la France occupée. La dualité institutionnelle ainsi rétablie entre domaine politique (attribué au CNI) et service de renseignement militaire (relevant du BCRAM), revenait à rendre plus complexes les envois des agents en France. Ceux-ci, qui avaient souvent des missions doubles (à commencer par Jean Moulin), recevaient des instructions et de Diethelm et de Passy. Il y avait parfois des contradictions ou des incohérences dans la définition de certaines missions. En outre, cette séparation théorique à laquelle de Gaulle demeurait attaché posait des

\footnotetext{
12. Manuel (A.), «Le BCRA de 1940 à $1944 »$, Les armées françaises pendant la seconde guerre mondiale 1939-1945, Paris, FEDN, 1986, p. 198.

13. Ceux-ci ne créèrent une branche " action " qu'en 1943 en Afrique du Nord, également avec l'aide alliée.

14. Service historique de l'armée de terre, 3 K 29 III, plage 1, entretien avec le colonel Passy.
} 
problèmes délicats dans la France occupée, en particulier dans les relations avec la Résistance qui n'opérait pas toujours de distinction entre action et renseignement de même qu'entre action politique et action paramilitaire. Ainsi, le BCRAM recevait souvent, sans le solliciter, du renseignement à caractère politique. Francis Louis Closon (1910-1998), directeur des services financiers de la France libre et qui s'occupait des fonds secrets du BCRAM a témoigné de cette évolution: "Le rôle du BCRAM, celui d'un "deuxième bureau" très élargi, bien que militaire, par la force des choses côtoyait dans une limite difficile à définir les affaires politiques ${ }^{15}$. "

L'arrivée à Londres de Pierre Brossolette (1903-1944) - ancien membre du réseau du Musée de l'homme et de la Confrérie Notre-Dame - en avril 1942, constitua une étape fondamentale dans l'évolution des services spéciaux gaullistes, les tirant de leur situation ambiguë ${ }^{16}$. Brossolette devint l'adjoint de Passy à la tête du BCRAM et fit, selon ses propres termes, l' ' éducation politique ${ }^{17} \gg$ du commandant Dewavrin. Les deux hommes réfléchirent à la réorganisation de l'action et du renseignement: "Dans les circonstances où nous nous trouvions à cette époque, vouloir séparer le renseignement et l'action politique du renseignement et de l'action militaire était une conception purement théorique. Il n'y avait pas de renseignements purement militaires et des renseignements politiques; pas davantage d'action purement militaire et d'action politique: renseignements militaires et renseignements politiques, action militaire et action politique, action et renseignements mêmes se pénétraient mutuellement de la façon la plus étroite $^{18}[\ldots] »$, écrivit plus tard Passy. Ils proposèrent en mai 1942 la création d'une "section politique " au sein du BCRAM ${ }^{19}$ qui devint à cette occasion BCRA - celui-ci perdant le " $M$ " militaire et se dotant de cette nouvelle section 20 , appelée «NM» (pour «non militaire»). Ainsi, le $2^{\mathbf{e}}$ bureau originel devint un organe mixte de renseignement et d'action aux attributions militaires et politiques en rupture complète avec les usages des services de renseignements traditionnels.

15. Closon (F.-L.), Le temps des passions. De Jenn Moulin à la Libération 1943-1944, Paris, Presses de la Cité, 1974, p. 31.

16. Cf. Piketty (G.), Pierre Brossolette. Un héros de la Résistance, Paris, Odile Jacob, 1998.

17. "J'ajoute qu'au cours de notre mission, il a fait mon éducation politique, domaine où je ne connaissais rien avant la guerre" (propos du colonel Passy recueillis par O. Wieviorka, Libération, 5 février 1998).

18. Colonel Passy, Solvenirs..., op. cit., p. 90-91.

19. Note du 30 mai 1942 reproduite dans Colonel Passy, Souzenirs..., op. cit., p. 92-94.

20. Sur l'activité de cette section, cf. Pierre-Bloch (J.), Le temps d'y penser 'ncore, Paris, JeanClaude Simoën, 1977, p. 213-220 et du même auteur : Le vént soufflè sur l'histoire. Témoignagés et documents inédits, Paris, SIPEP, 1956. 


\section{Le contrôle républicain et civil du renseignement militaire à l'heure d'Alger}

Cette évolution qui allait à l'encontre du principe républicain de séparation stricte des domaines militaire et politique fut vivement contestée par la Résistance intérieure. En outre, en 1943, dans un contexte marqué par la lutte entre de Gaulle et Giraud, se posa la question de la réunification de l'armée et donc également de celle des services de renseignement. A cette occasion apparut une nouvelle difficulté, laissée en suspens depuis la défaite. Sous la III ${ }^{\text {e }}$ République, les services de renseignement étaient rattachés au ministère de la Guerre et ils échappaient à ce titre à toute forme de contrôle civil, même s'ils restaient soumis en dernier lieu à l'autorité politique par le biais du ministre. Or, dès 1943, la rivalité entre de Gaulle et Giraud amena le premier à opérer le rattachement des services à une autorité collégiale où le pouvoir civil était représenté. En effet, le 4 octobre 1943, deux décrets étaient signés en séance du Comité français de libération nationale (CFLN) par les deux coprésidents, de Gaulle et Giraud. Les textes créaient un comité d'action en France, appelé Comidac, ayant désormais autorité sur les services spéciaux dont la fusion était par ailleurs demandée. Le Comidac était composé du président du CFLN et du commandant en chef ainsi que d'une troisième personne, le commissaire à l'Intérieur, c'est-à-dire d'un membre civil ayant rang de ministre. Le général de Gaulle parvint avec une intelligence politique qui faisait défaut au général Giraud à évincer celui-ci de son poste de coprésident le 9 novembre.

La personnalité du nouveau commissaire à l'Intérieur, Emmanuel d'Astier de la Vigerie (1900-1969), l'un des fondateurs du mouvement LibérationSud, nommé le jour même où Giraud abandonnait tout rôle politique pour devenir commandant en chef, joua un rôle important dans le sens d'un contrôle civil sur les services. De Gaulle et son ministre d'Astier contrôlaient désormais le Comidac et, par son intermédiaire, les services spéciaux. La nomination de d'Astier réactiva le conflit latent depuis l'Affaire Dreyfus entre fonctionnaires civils et fonctionnaires militaires du renseignement. En outre, la rivalité entre la Résistance intérieure, représentée par d'Astier et la France libre, incarnée par le BCRA, atteignit à ce moment son apogée. Le nouveau ministre de l'Intérieur avait en effet une conception très large de sa nouvelle fonction: «[...] Cela signifiait la tâche de coordination de la Résistance, le contrôle, que j'ai plus ou moins obtenu, de tous les services secrets, et les négociations internationales ${ }^{21}[\ldots]$ »Dans un rapport rédigé par d'Astier à la fin du mois de janvier 1944, celui-ci analysait clairement la situation au moment de sa nomination: «Les services spéciaux chargés de l'exécution des moyens sur la France étaient un organisme dont l'accès était fermé à toute autorité autre que celle de quelques officiers, souvent capables,

21. Crémieux (F.), Entretiens avec Emmanuel d'Astier, Paris, Pierre Belfond, 1966, p. 113. 
mais n'obéissant que trop souvent à des vues personnelles et qui ne suivaient pas une ligne de conduite strictement établie par un organisme gouvernemental ${ }^{22}$. "

Peu de temps après sa nomination, d'Astier obtint du général de Gaulle la création d'un nouvel organe, la Direction générale des services spéciaux (DGSS) dont le but était à la fois de mener à bien la fusion entre le BCRA et les services spéciaux de l'armée d'armistice (DSR-SM), repliés en Afrique du Nord en novembre 1942, et d'asseoir définitivement la tutelle civile sur l'ensemble des services. Le décret du 20 novembre 1943 instituant la DGSS prévoyait que le chef des services spéciaux serait désormais un civil et que le poste de directeur général technique adjoint, "chargé de l'aspect politique de l'action en France ${ }^{23}$ » appartiendrait au commissariat à l'Intérieur. Par ailleurs, d'Astier commença à démembrer le BCRA, malgré l'opposition commune de Passy et de Jacques Soustelle ${ }^{24}$, en obtenant le 10 janvier 1944 que la section "NM » - la section politique créée par Brossolette et Passy soit désormais intégrée au commissariat à l'Intérieur. Le même jour, d'Astier fit acclamer cette nouvelle organisation des services spéciaux par les députés de l'Assemblée consultative provisoire. En présence du général de Gaulle, Emmanuel d'Astier déclara devant l'hémicycle: "Qu'avons-nous voulu ? Que les services spéciaux soient un outil de gouvernement ; qu'ils cessent d'être l'outil d'une caste militaire ou d'un clan politique ? Or, je peux le dire ici, cela est fait. Désormais, les services spéciaux ne seront qu'un outil de Gouvernement [...] (Très bien 25 !) » Une motion fut ensuite votée à l'unanimité, demandant l'unification des services "sous l'autorité du Commissariat à l'intérieur et en accord étroit avec la délégation de la Résistance française à Alger 26. " L'Assemblée et le ministre de l'Intérieur communiaient ainsi dans un même esprit résistant et républicain. A propos d'un conflit annexe entre Passy et d'Astier, celui-ci, qui avait mis sa démission dans la balance, obtint du général de Gaulle en avril 1944 la mutation du fondateur du BCRA à l'état-major FFI auprès de Koenig. A l'approche de la libération de la France, le général de Gaulle opérait ainsi un

\footnotetext{
22. Archives nationales, F 1a 3727, "Rapport sur l'activité du commissaire à l'intérieur du 8 novembre [1943] au 20 janvier [1944] », p. 1.

23. Archives nationales, 72 AJ 408, fonds Emmanuel d'Astier, article 2 du décret "fixant l'organisation des services de renseignements et d'action, texte proposé par le commissaire à l'intérieur en vue de son inscription à l'ordre du jour de la séance du 16 novembre [1943]. "Ce décret fut signé par le général de Gaulle le 20 novembre.

24. Pour plus de précisions sur ce point, nous renvoyons à notre étude : Laurent (S.), « The Free French Secret Services: Intelligence and the Politics of Republican Legitimacy ", Intelligence and National Security, 4, 2000.

25. Journal officiel de la République française. Débats de l'assemblée consultative provisoire, séance du 10 janvier 1944, p. 15. Propos partiellement reproduits dans Cerf-Ferrière (R.), L'Assemblée consultatize z'lue de mon banc. Nozembre 1943-juillet 1944, Paris, Editeurs français réunis, 1974, p. 225. 26. Journal officiel de la République française. Débats de l'assemblée consultatize prozisoire, séance du 10 janvier 1944, p. 15.
} 
choix politique en maintenant au gouvernement un responsable important de la Résistance intérieure.

La question de l'amalgame entre giraudistes et gaullistes qui se posa pour l'ensemble des armées combattantes en $1944^{27}$ opposa ainsi dès 1943 les services de l'armée d'armistice, la DSR-SM commandée par le colonel Rivet (1883-1958), au BCRA. Les conditions de cet amalgame étaient tout à fait particulières. En premier lieu, la DSR-SM se posait logiquement comme l'institution de l'armée traditionnelle succédant au SR-SCR dont elle possédait le personnel, le savoir-faire et les traditions. Face à elle, le BCRA légitimait son existence par un tout autre discours reposant sur l'urgence de la situation en 1940. Par ailleurs, le commandement de la DSR-SM était essentiellement composé d'un corps d'active ${ }^{28}$, alors que le BCRA comptait dans ses rangs avant tout des civils et des réservistes, à l'exception de son chef. Enfin, la conséquence logique de ces caractéristiques différentes était de poser ces deux institutions sur des plans politiques opposés: la DSR-SM incarnait une culture politique conservatrice, bien qu'elle s'en défendît par son discours sur "l'apolitisme", et le BCRA associait des individus aux sensibilités beaucoup moins homogènes. L'exploitation du thème du BCRA " cagoulard " par des milieux londoniens hostiles au général de Gaulle est sans fondement: si Maurice Duclos (1906-1981) fut en effet membre du Comité secret d'action révolutionnaire (CSAR) et Pierre Fourcaud (18981998) peut-être également, ces cas demeurèrent des exceptions dans un organisme inclinant plutôt à gauche.

La fusion entre les deux maisons fut cependant accomplie au forceps et il revint à Jacques Soustelle (1912-1990), qui avait été nommé directeur général de la DGSS à sa création, de la mener à bien. La Direction générale des études et de la recherche (DGER) qui lui succéda et dont Passy, revenu en grâce, avait pris la tête en mai 1945 poursuivit cette tâche ardue. Dans le même temps où il préparait une réorganisation profonde des services de renseignement, la DGER subissait les attaques régulières des résistants à l'Assemblée consultative provisoire $^{29}$. En septembre 1945, les services de la défense nationale publièrent sous le sceau du gouvernement provisoire un communiqué de presse: "La DGER joue un rôle d'intérêt national à

27. Cf. Delmas (J.), «L'amalgame: Forces françaises de l'intérieur 1re armée ", Les armées françaises pendant la seconde guerre mondiale..., op. cit., et du même auteur: "La renaissance de l'armée française ", La France de 1945. Résistances retours renaissances, Caen, Presses universitaires de Caen, 1996.

28. De nombreux officiers de réserve avaient été mobilisés en 1939 au SR-SCR et certains demeurèrent dans leur fonction pendant toute la durée de la guerre. En outre, des civils, hommes et femmes, travaillèrent soit comme " honorables correspondants" soit comme agents pour de nombreux réseaux de renseignement relevant de la DSR-SM. Cependant, l'encadrement était composé essentiellement d'officiers de métier.

29. En décembre 1944 celles de P. Copeau et en mars 1945 celles de P. Lebrun. 
l'extérieur de nos frontières; elle n'en joue aucun à l'intérieur ${ }^{30}$. " Cependant, les critiques ne désarmèrent pas et en octobre 1945, l'assemblée des présidents et délégués des comités de Libération, adopta une motion votée à l'unanimité demandant «la suppression de toute police politique incontrôlée telle que la DGER ${ }^{31}$ ". Une nouvelle fois, comme au temps de l'offensive de d'Astier contre l'organisation Brossolette-Passy, les règlements de compte entre la Résistance intérieure et celle de Londres reposaient sur un discours républicain réclamant la dissociation des missions entre policiers et militaires à l'occasion du retour à la paix.

\section{La création du SDECE : une victoire républicaine à la Pyrrhus ?}

Passy parvint néanmoins à faire signer en conseil des ministres, le 28 décembre 1945, un décret rattachant le Service de documentation extérieure et de contre-espionnage (SDECE) à la présidence du Conseil ${ }^{32}$. Ce nouveau dispositif, qui demeura valide jusqu'en 1966 33 , retirait le service de renseignements de la tutelle du ministère de la Guerre pour la confier au pouvoir civil. A l'évidence, Passy avait tiré les leçons de l'organisation du dispositif de renseignement anglais. En outre, le nouveau statut du SDECE entériné par le général de Gaulle contentait les aspirations des résistants telles qu'elles avaient été formulées depuis 1943. Comme en 1899, le service de renseignements militaire qu'était le SDECE n'avait d'autorité qu'à l'extérieur du territoire national. Le renseignement sur le territoire relevait d'un service de police, la Direction de la surveillance du territoire (DST), créée en novembre 1944 et confiée à Roger Warin (1912-1997) - «Wybot » dans la Résistance - qui avait servi en 1941 dans les services de renseignement de l'armée d'armistice au Bureau des menées antinationales de Marseille, puis en 1941-1942 au BCRA ${ }^{34}$.

Si la nouvelle organisation du dispositif de renseignement désormais rattaché à la présidence du Conseil avait été accomplie dans le sens souhaité par la Résistance, l'amalgame entre le SR-SCR et le BCRA fut bien réel et les "spécialistes" - pour reprendre le terme $d^{\prime}$ André Manuel - demeuraient majoritaires aux postes de direction du SDECE. Certes, le commandant

30. Le Monde, 23-24 septembre 1945, p. 2.

31. Le Monde, 28-29 octobre 1945, p. 2.

32. Cf. Service historique de l'armée de terre, $6 \mathrm{P} 1$, "Relevé des projets de lois et de décrets adoptés au conseil des ministres du 28 décembre 1945 ". Le décret non publié fut considéré comme promulgué le 4 janvier 1946.

33. A la suite de l'affaire Ben Barka, le général de Gaulle, revenant aux usages de la III République, décida par décret du 2 janvier 1966 de confier la tutelle du SDECE au ministre des Armées.

34. D'après Passy, c'est Wybot qui proposa de transformer le sigle BCRAM en BCRA (Colonel Passy, Sowvenirs..., op. cit., p. 29). Celui-ci ne fit qu'un passage rapide au contre-espionnage du BCRA avant de rejoindre une unité combattante. 
Paillole (né en 1905), officier du contre-espionnage depuis 1935 et chef du contre-espionnage depuis 1940, hostile à la réorganisation entamée en 1943, décida de quitter les services en novembre 1944. Cependant de très nombreux officiers du SR-SCR, qui avaient commencé à y travailler à la fin des années 1930, occupèrent des fonctions stratégiques : le colonel MauriceHenri Dumont (né en 1911), occupa le poste important de directeur de la recherche, le capitaine Roger Lafont (1897-1952) dirigea le contreespionnage, le capitaine de vaisseau Henri Trautmann prit à sa charge les services de recrutement et $d$ 'instruction et le commandant Gustave Bertrand s'occupa des services techniques du SDECE.

La nouvelle organisation ne satisfaisait pas pourtant toute la Résistance. Le 30 décembre 1945, lors des débats budgétaires à l'Assemblée constituante, Emmanuel d'Astier lança une attaque très violente contre le SDECE, demandant que les crédits ne lui soient accordés que pour trois mois ${ }^{35}$. Vincent Auriol, président de l'Assemblée constituante, apporta de nouvelles garanties : "Le nouveau service a une mission exclusive de toute recherche sur des territoires relevant de la souveraineté française ; il est contrôlé par un comité interministériel présidé par le président du gouvernement; son budget sera soumis à la commission spéciale de défense nationale ${ }^{36}$. "Ce comité, qui ressemblait dans son principe au Joint Intelligence Committee institué à Londres en 1936, était composé des ministres d'Etat, de ceux des Affaires étrangères, de l'Intérieur, des Armées, des Finances, de l'Economie et de l'Armement. L'amendement d'Astier fut repoussé par 521 voix contre 44.

\section{La nouvelle position du renseignement dans l'espace public : l'affaire Passy}

L'affaire Passy, qui survint en 1946-1947, est un témoignage des nouvelles relations établies entre les services de renseignements et le pouvoir ainsi que de la place nouvelle prise par le renseignement dans l'espace public. Un mois après le départ du général de Gaulle ${ }^{37}$, Passy quitta sa fonction de directeur général du SDECE. Le nouveau président du Conseil, Félix Gouin, nomma à sa place, le 22 février 1946, un ancien collaborateur de Marx Dormoy, Henri Ribière, l'un des fondateurs de Libération-Nord, député socialiste de l'Allier à l'Assemblée constituante. Avec la nomination de Ribière, premier homme politique nommé à la tête des services secrets militaires, le successeur du général de Gaulle marquait sa volonté de parachever l'œuvre de la Résistance.

35. Le Monde, ler janvier 1946, p. 3.

36. Ibid.

37. Cf. Elgey (G.), Histoire de la IVe République. La République des illusions 1945-1951, Paris, Fayard, 1993, p. 79-129. 
Dans la nuit du 6 au 7 mai 1946, l'agence France-Presse publia un communiqué indiquant que Passy et certains de ces collaborateurs avaient été arrêtés ${ }^{38}$. Le lendemain, Gaston Defferre, secrétaire d'Etat à l'Information, déclara à la sortie du conseil des ministres que soixante jours $\mathrm{d}^{\prime}$ arrêts de forteresse avaient été infligés au colonel Passy ajoutant, sans plus de précisions, que l'affaire pour laquelle cette sanction avait été prise était antérieure au référendum du $5 \mathrm{mai}$ et qu'elle n'avait pas été révélée auparavant afin de ne pas faire preuve de malveillance politique ${ }^{39}$. Deux jours après, des fuites parues dans Le Monde révélèrent que la sanction avait été prise parce que Passy ne parvenait pas à justifier l'emploi et l'origine de plusieurs dizaines de millions de francs qui avaient été retrouvés depuis la passation de comptabilité ${ }^{40}$. L'article indiquait en outre qu'une enquête administrative interne à l'armée, diligentée par Ribière, était en cours sur les activités du colonel Passy. Il ne fut ensuite plus question de cette affaire jusqu'au mois de juillet 1946, lorsque Emmanuel d'Astier, devenu député progressiste d'Ille-et-Vilaine, demanda, lors d'une séance de questions au gouvernement, qu'une commission parlementaire restreinte examine les conclusions de l'enquête administrative ${ }^{41}$.

L'affaire sortit pourtant des cercles gouvernementaux lorsque le conseil des ministres annonça le 30 août 1946 qu'un "conseil d'enquête " militaire allait être réuni afin de se prononcer sur l'exclusion de l'armée du colonel Passy ${ }^{42}$. Dès le lendemain, celui-ci intervint pour la première fois publiquement en transmettant un communiqué à l'AFP. Il présentait son système de défense qui, jusqu'à la fin, ne varia pas : il affirmait n'avoir rien détourné à son profit personnel, demandait à ce que l'affaire sorte de son cadre administratif pour être transmise à la justice afin qu'une instruction libre " sans pièces secrètes " puisse être menée. Enfin, il affirmait s'être conformé aux usages des services de renseignement: «[...] Si l'utilisation de fonds mis à ma disposition pouvait sembler inopportune aux dirigeants actuels, il ne peut s'agir que d'une divergence de conception, la mienne étant rigoureusement conforme à la conception habituelle de tous les SR en France et à l'étranger ${ }^{43}$. " La réaction de Passy surprit le gouvernement et incita celui-ci à réagir à nouveau. Devant une centaine de journalistes, Pierre-Henri Teitgen, ministre de la Justice du gouvernement Bidault, rendit publics les résultats de l'enquête administrative menée par Henri Ribière. Teitgen indiqua que Passy avait fait des aveux concernant l'existence de dépôts clandestins d'argent

38. Le Monde, 7 mai 1946, p. 5. Pour les réactions du général de Gaulle, cf. Guy (C.), En écoutant de Gaulle. Journal 1946-1949, Paris, Grasset, 1996, p. 64 et Mauriac (C.), Le' temps immobile. Aimer de Gaulle, Paris, Grasset, 1978, p. 287.

39. Le Monde, 8 mai 1946, p. 8.

40. "L'affaire de la DGER ", Le Monde, 10 mai 1946, p. 1.

41. "L'affaire Passy ", Le Monde, 25 juillet 1946, p. 4.

42. Le Monde, 30 août 1946, p. 3.

43. Le texte du communiqué fut publié dans Le Monde, 31 août 1946, p. 4. 
non inscrits dans la comptabilité, mais le ministre MRP s'empressait de dégager immédiatement la responsabilité du général de Gaulle ${ }^{44}$. Enfin, répondant à Passy, il lui semblait que l'affaire ne pouvait avoir de suites judiciaires : «Cela semble difficile au ministre en raison du caractère secret des faits en cause » commentait Le Monde.

L'ensemble de la presse s'empara de l'affaire et lui donna alors un écho considérable. La presse gaulliste défendait Passy 45 , alors que les résistants l'attaquaient et que la gauche radicale 46 critiquait les méthodes du gouvernement MRP, notamment celles du ministre des Armées Edmond Michelet et celles du garde des Sceaux ${ }^{47}$. Passy réagit à la conférence de presse dans un long article intitulé "Ma défense ", publié le 12 septembre 1946 dans Paris-Presse. Il reconnaissait l'existence de dépôts clandestins, se posait en fidèle du général de Gaulle ${ }^{48}$ tout en indiquant clairement qu'il souhaitait le tenir à l'écart de cette affaire. Il réitérait l'argument technique invoqué quinze jours plus tôt dans le communiqué de l'AFP : "C'est sur le plan SR et sur ce plan exclusivement que mon action doit être jugée ${ }^{49}$. » Il terminait en politisant le débat, jouant habilement sur le fossé qui commençait à grandir entre le MRP et le général de Gaulle ${ }^{50}$ : «Puisse le pays ne pas penser, demain, que j'ai été sacrifié sur l'autel du tripartisme par les dirigeants du "parti de la fidélité du bout des lèvres ${ }^{51 " . ~ " ~ P e u ~ d e ~ t e m p s ~ a p r e ̀ s ~}{ }^{52}$, il déposa plusieurs pourvois auprès du Conseil d'Etat en vue d'annuler son renvoi devant le " conseil d'enquête " ainsi qu'une plainte contre $X$ pour "arrestation arbitraire et séquestration $^{53}$ ». Entre-temps le MRP avait réagi par le biais de Teitgen dont le chef de cabinet, Michel de Boissieu, était allé à Colombey pour demander au général de Gaulle de modérer les réactions de Passy qui fragilisaient, d'après le ministre ${ }^{54}$, le gouvernement. En avril 1947, Passy commença la publication de ses Mémoires dans Paris-Presse. Malgré la publication, le 3 juin 1947, par le

44. Le Monde, 8-9 septembre 1946, p. 5.

45. Cf. Lescure (M.), "L'homme à abattre ", La France libre, 10 septembre 1946, p. 3.

46. Cf. Stéphane (R.), "Pas de scandale dans les arrêts du colonel Passy", Libération, 10 septembre 1946.

47. Cf. Quilici (F.), "La véritable affaire Passy", La bataille politique et littéraire, 11 septembre 1946, p. 1-2 et la série d'articles de M. Rucart dans La Dépêche de Paris (17-19 septembre, 21-24 septembre 1946).

48. Sur ce point, cf. Lachaise (B.), "Contestataires et compagnons. Les formes de l'engagement gaulliste », Vingtième siècle, 60, 1998.

49. "Ma défense », Paris-Presse, 12 septembre 1946.

50. En 1945-1946, le MRP tentait de s'identifier au parti du général de Gaulle et y parvint auprès de l'électorat jusqu'à l'émergence du différend constitutionnel entre le général et le MRP le 13 octobre 1946 (cf. Letamendia (P.), Le mouvement républicain populaire. Histoire d'un grand parti français, Paris, Beauchesne, 1995, p. 70-75). Voir également sur ce point Charlot (J.), Le gaullisme d'opposition 1946-1958. Histoire du gaullisme, Paris, Fayard, 1983, p. 51-64.

51. Ibid., p. 2.

52. Cf. Le Monde, 14 septembre 1946, p. 4 et Combat, 13 octobre 1946, p. 4.

53. Le Monde, 19 décembre 1946, p. 5.

54. Cette visite eut lieu le 9 octobre 1946, voir Guy (Cl.), En écoutant de Gaulle..., op. cit., p. 134. 
quotidien communiste $\mathrm{Ce}$ Soir ${ }^{55}$ de l'enquête administrative de Ribière qui précisait le contenu des accusations portées contre Dewavrin et le rejet des pourvois par le Conseil d'Etat en juillet, l'affaire sombra dans l'oubli assez rapidement. Le gouvernement Ramadier ne parvint pas à obtenir des officiers du «conseil d'enquête " que celui-ci tienne ses réunions et refusa comme le cabinet précédent de traduire Dewavrin devant la justice ${ }^{56}$. La stratégie de défense de Passy, qui avait tenté de porter l'affaire sur le plan judiciaire et politique tout en maintenant un argumentaire technique, avait parfaitement fonctionné. Une fois encore, il sut tirer plus tôt que d'autres les conséquences de la situation nouvelle des services secrets au sein de la République.

A l'occasion de la guerre, les services de renseignement sont donc sortis du «pré carré " militaire : rattachés au pouvoir civil à partir de 1943, ils font désormais l'objet de discussions publiques soit au Parlement, soit dans la presse; on en parle désormais aux plus hauts niveaux comme d'une "administration essentielle à $l^{\prime} E t_{a} t^{57}$ », pour reprendre les paroles mêmes du général de Gaulle en 1946. Ces conditions nouvelles pesèrent fortement sous les régimes suivants, en particulier lors de la guerre d'Indochine et d'Algérie. La défaite militaire et la division des services de renseignements ont joué un rôle considérable dans cette évolution. L'apport de deux individus, Passy et Brossolette, ignorant tout de cette spécialité militaire, a également été décisif. Brossolette a su tirer très tôt de la guerre clandestine les leçons qui s'imposaient et il est parvenu à faire accepter au général de Gaulle une politisation du BCRA que toute sa formation d'officier réprouvait. Emmanuel d'Astier, fortement appuyé par l'Assemblée d'Alger a été le principal agent du contrôle civil sur les services spéciaux. Il ne faut pas négliger également l'influence du modèle que constituèrent l'Intelligence service et le SOE aux yeux de Passy lorsqu'il créa le SDECE. Les profondes réformes des services entre 1943 et 1945 ont imposé un nouveau dispositif de renseignement à la Libération, mais il a fallu plusieurs années pour que les officiers s'habituent à travailler dans une maison dirigée par un civil, soumis à l'autorité du président du Conseil et pour effacer bien des cicatrices laissées par les luttes ayant opposé les deux maisons entre 1940 et 1945. Les exigences du secret pour les services de renseignements sont restées aussi fortes qu'auparavant mais ces officiers qui avaient appris que les "services secrets ne défilaient pas sous l'arc de Triomphe ", durent désormais accepter d'être soumis au regard inquisiteur des assemblées et de la presse, situation sans précédent pour ces officiers de l'ombre.

55. De longs extraits parurent dans le Monde: «La publication du rapport administratif établi l'an dernier par M. Ribière ramène l'attention sur l'affaire Passy ", Le' Monde, 3 juin 1947, p. 4.

56. Celui-ci quitta l'armée à la fin de l'année 1948.

57. Propos tenus par le général de Gaulle le 20 mai 1946, Guy (Cl.), En écoutant de Gaulle..., op. cit., p. $68-69$. 